\title{
Suboptimal behaviour and knowledge regarding overnight glycaemia in adults with Type 1 diabetes is common
}

\section{Authors' Names and Affiliations}

Christina R. Larsson ${ }^{1,2}$, Andrzej S. Januszewski ${ }^{2}$, Rachel T. McGrath ${ }^{3}$, Johnny Ludvigsson ${ }^{1,4}$, Anthony C. Keech ${ }^{2}$, Richard J MacIsaac ${ }^{5}$, Glenn M Ward ${ }^{5}$, David N. O’Neal ${ }^{5}$, Gregory R. Fulcher ${ }^{3}$, Alicia J. Jenkins ${ }^{2,5}$

1. Faculty of Medicine, Linköping University, Sweden, SE-581 83 Linköping

2. NHMRC Clinical Trials Centre, The University of Sydney, Camperdown, NSW 2050, Australia

3. Department of Diabetes, Endocrinology and Metabolism. The University of Sydney, Northern Clinical School, Royal North Shore Hospital, Royal North Shore Hospital, St Leonards, NSW 2065

4. Department of Clinical and Experimental Medicine, IKE, Linköping University, Sweden, SE-581 83 Linköping

5. Department of Diabetes and Endocrinology, St. Vincent's Hospital Melbourne and University of Melbourne, Fitzroy VIC 3065, Australia

\section{Positions}

C.R.L. - Medical Student; A.S.J. - Senior Research Fellow; R.T McG - Research Fellow; JL Senior Professor of Paediatrics; ACK - Professor and Deputy Director NHMRC Clinical Trials Centre, RMacI - Professor and Director of Endocrinology, St Vincent's (Melbourne), GW Professor, Director of Diabetes Services, St Vincent's Hospital (Melbourne), DNO - Associate Professor, Endocrinologist, G.R.F - Clinical Professor of Medicine, Senior Staff Specialist; A.J.J. Professor, Diabetes and Vascular Medicine; Endocrinologist.

\section{Corresponding Author}

Professor Alicia Jenkins

NHMRC Clinical Trials Centre

Level 6, Medical Foundation Building

92 - 94 Parramatta Road, Camperdown, NSW, 2050

Telephone: +61 29562 5000; Facsimile: +61 295625090

Email: alicia.jenkins@,ctc.usyd.edu.au

Word counts: Abstract: 250; Main Text 2514

Tables: 3, Figure: 1

This is the author manuscript accepted for publication and has undergone full peer review but has not been through the copyediting, typesetting, pagination and proofreading process, which may lead to differences between this version and the Version of Record. Please cite this article as doi: 10.1111/imj.13798

This article is protected by copyright. All rights reserved. 
Keywords: Type 1 Diabetes, Nocturnal Hypoglycaemia, Hyperglycaemia, Self-Management, Diabetes Education

This article is protected by copyright. All rights reserved. 


\section{Introduction}

Most adults with Type 1 diabetes (T1D) spend HB-hours a year with their diabetes care-team, therefore glucose self-management is essential. Hypoglycaemia is common, often feared, and limits achievement of near-normoglycaemia that decreases chronic complications ${ }^{1-3}$. An adult with T1D typically has two mild hypoglycaemia events weekly and one severe hypoglycaemia (SH) event, defined as that requiring assistance from another person for recovery, per year ${ }^{4}$. Over half of hypoglycaemia events occur overnight and are slept through ${ }^{5,6}$. Nocturnal hypoglycaemia (NH) can be unrecognised as sleep reduces counter-regulatory responses ${ }^{7}$, and $\mathrm{NH}$ can cause seizures, cardiac arrhythmias, and death ${ }^{8}$. NH risk can be reduced by flatter profile insulins ${ }^{9}$, continuous glucose monitoring $(\mathrm{CGM})^{5}$, insulin pumps, particularly with a low glucose (insulin) suspend function ${ }^{10}$, and patient education ${ }^{11}$. Identification of those who will benefit from education is key to enhance patient well-being and reduce carer and healthcare system burden. There are limited data regarding patient knowledge and behaviour related to $\mathrm{NH}$ prevention and care ${ }^{12}$.

We developed a questionnaire for T1D adults to assess glucose self-management, particular that related to overnight BG. We believe this is the first such Australian study.

\section{Subjects and Methods}

The study was approved by Human Research Ethics Committees: Northern Sydney Local Health District, (\#RESP/15/226) and St Vincent's Health (SVH), Melbourne, (\#LRR137/15). Implied consent was obtained at Royal North Shore Hospital (RNSH) by survey completion and written informed consent at SVH.

Subjects were aged e 18 years with T1D or latent autoimmune diabetes of adults (LADA). All T1D or LADA adults attending RNSH or SVH diabetes outpatients during the 3-month study period were invited to participate (by CRL, RMcG or AJJ) whilst in the waiting room. After consenting they were provided with the paper questionnaire. Exclusion criteria were: age $<18$ years, pregnancy, 
other diabetes types and inadequate English. Participants were asked to complete the survey whilst in-clinic. The 16-question survey (available free from the corresponding author) included: demographics, usual glycaemia-related behaviour, self-assessment of glucose management knowledge, response to six hypothetical pre-bed $\mathrm{BG}$ readings $(4,8,11,15,18 \mathrm{mmol} / 1$ (with moderately high $(1.0 \mathrm{mmol} / \mathrm{L})$ blood ketones) and $20 \mathrm{mmol} / \mathrm{L})$ and desire for further diabetes education. Impaired hypoglycaemia awareness (IHA) status was based on self-report (full, partial or unaware) of hypoglycaemia awareness. Questionnaire completion time was recorded in a random subset. Recent (within 3-months) HbA1c results were retrieved from medical records. Responses to hypothetical BG-levels were categorised (by endocrinologists AJJ, GRF) as safe, unsafe or suboptimal. Suboptimal/unsafe responses included: not eating if pre-bed BG was d4mmol/L, not taking insulin and/or not rechecking $\mathrm{BG}$ overnight for pre-bed $\mathrm{BGe} 15 \mathrm{mmol} / \mathrm{L}$; and/or not testing ketones again for pre-bed BGe $18 \mathrm{mmol} / \mathrm{L}$; eating without taking insulin for pre-bed BGe $11 \mathrm{mmol} / \mathrm{L}$ and taking excess insulin to lower pre-bed BG levels. Excess insulin was defined as e $10 \%$ that calculated to correct the BG to $6 \mathrm{mmol} / \mathrm{L}$ using the 'rule of 100 ' $(100 /$ total daily insulin dose, which estimates the BG lowering (in $\mathrm{mmol} / \mathrm{l}$ ) by 1 unit of insulin). The treating doctor was alerted if answers indicated potentially unsafe behaviours and (irrespective of answers), if participants desired education.

Data were managed in EXCEL (Microsoft, Redmond, Washington: Microsoft 2003), with random validation of $10 \%$ of data-entry and analysed in STATISTICA for Windows (StatSoft, Inc. (2012): STATISTICA (version 12, Oklahoma, USA) and ACCord (Analysis of Censored and Correlated Data, Boffin Software, version 2.0.10, Ryde, NSW). Normal distribution of continuous variables was assessed (Kolmogorov-Smirnov test) and descriptive statistics, Chi-square tests, t-tests or Welch t-tests and logistic regression were used. Significance was taken at $\mathrm{p}<0.05$. 


\section{Results}

Table 1 shows demographics of all 205 participants, and by Continuous Subcutaneous Insulin Infusion (CSII) and Multiple Daily Injections (MDI) use, by IHA status and by desire for further education. Over $90 \%$ of eligible patients participated; with $98 \%$ having T1D and 2\% LADA. Seventy one percent attended RNSH (31\% on CSII); $53 \%$ of SVH participants used CSII, $(p=0.003)$. Apart from CSII use RNSH and SVH subjects and results did not differ significantly, hence data were grouped. The survey took 10-15 minutes, and 8\% of surveys were incomplete, with missing responses averaging $\mathrm{H} 6 \%$ of questions.

Self-reported home BG monitoring habits are summarised in Table 2. The mean (SD) frequency of BG tests was $5.4(2.7)$ /day, being higher in CSII vs. MDI users $(\mathrm{p}=0.01)$, but similar by IHA status or education desire. About one third of patients said that they never test their overnight BGs and 9\% said they test every night. Self-reported BG testing frequency correlated inversely with concurrent HbA1c $(r=-0.17 ; p=0.02)$ overall, in CSII-users $(r=-0.29 ; p=0.01)$ and in subjects reporting full hypoglycaemia awareness $(\mathrm{r}=-0.20 ; \mathrm{p}=0.02)$.

Thirty-one percent of participants reported not having in-date ketone test-strips at home, with no differences by insulin delivery mode, IHA status, nor education desire (all $\mathrm{p}>0.05$ ).

In general, BG targets (Table 2) were similar in CSII and MDI users; except CSII-users targeted a lower bed-time BG (7.1 (1.2) vs 7.7 (1.4), p=0.005) and subjects desiring further education targeted a higher pre-breakfast $B G, p=0.03$. There were no significant differences by IHA status.

Self-reported severe hypoglycaemia (SH) frequency, (Table 2), was similar in all groups except for daytime SH being more common with CSII use and with IHA. The IHA group also had more frequent ( $>3 /$ week) daytime hypoglycaemic episodes (experienced by $28 \%$ of those with IHA vs. $14 \%$ with intact hypoglycaemia awareness).

This article is protected by copyright. All rights reserved. 
After waking with a $\mathrm{NH}, 71 \%$ of subjects reported they would test their BG pre-treatment. Only $44 \%$ reported testing soon after $\mathrm{NH}$ treatment, with most reporting not testing until morning. $\mathrm{NH}$ treatments are described in Figure 1. Patients were asked; "What determines when you test your blood glucose levels overnight?" The main reasons were if they "felt hypo" or were "awake for some other reason". Few would set an alarm to episodically test their overnight BG. Results were similar for CSII and MDI users and by IHA status ( $\mathrm{p}>0.05)$.

After day-time extra exercise, alcohol or illness, 48\%, 54\% and 48\% respectively reported they would not change their overnight BG care-plan. Only $\mathrm{HB} 0 \%$ said they would do so in all three situations (or two if they were a non-drinker, as were 15\%). There were no differences by CSII vs. MDI use. Only $79 \%$ reported feeling confident dealing with these situations; $21 \%$ felt partly or not confident. Thirty-two percent of patients desired additional diabetes education, including $70 \%$ of those with less than full confidence and $22 \%$ of those confident in these situations. Further education was desired by $44 \%$ of subjects reporting IHA vs. $25 \%$ with full hypoglycaemia awareness, $\mathrm{p}=0.006$. Education desire did not differ by insulin delivery modality nor by diabetes duration (both $\mathrm{p}>0.05$ ).

Table 3 presents percentages of patients suggesting safe behaviour at each hypothetical pre-bed BG. Of all suboptimal/unsafe answers for the six BG levels, $20 \%$ were designated so because of risk for hyperglycaemia/ketosis, $15 \%$ because of hypoglycaemia risk, and $65 \%$ because of risk for both. Generally, safer responses were suggested by CSII-users, those who BG-tested more frequently and targeted higher bed-time BG levels. Results did not differ by IHA status, CGM use nor education desire. In univariate logistic regression analysis, only MDI use and fewer BG tests/day associated with higher rates of suboptimal overnight glucose control answers; OR (95\% CI): 3.09 (1.64-5.81); $\mathrm{p}=0.0004$ and 1.17 (1.04-1.32); $\mathrm{p}=0.007$ respectively. Clinic site, gender, age, diabetes duration, 
HbA1c, IHA status and SH frequency did not influence unsafe/suboptimal responses $(p=0.73)$. In a multivariate logistic regression analysis adjusting for age, gender, diabetes duration, HbAlc and IHA status only MDI use and fewer BG tests/day remained independent predictors of suboptimal suggestions (OR (95\% CI): $2.8(1.4-5.4 ; \mathrm{p}=0.003$ and $1.17(1.03-1.33) ; \mathrm{p}=0.01)$.

\section{Discussion}

In this novel, clinically relevant study of T1D adults in two tertiary diabetes clinics, we demonstrated that it was feasible and time-efficient to use a self-administered survey to assess (selfreported) glucose care practices and identify patients with potentially risky behaviours related to overnight glucose management. Suboptimal practices, such as not having ketone test-strips, NH treatment and knowledge gaps were common.

It is recommended that adults with T1D test their BG levels H4-times daily, including episodically overnight, and have in-date ketone test-strips for sick-day management and other circumstances of hyperglycaemia. The Australian government subsidises test-strips for BG, but not ketones. Less frequent BG-testing is usually needed during CGM-use, though this relatively costly, user-pay system was infrequently and usually episodically used by survey participants, and 'flash glucose monitoring' of interstitial fluid ${ }^{13}$ was not available in Australia during this survey.

In our study the mean HbA1c was 7.8 (1.4)\% (61.3 (15.8) mmol/mol, less than that of Australian adults with T1D in the 2011 ANDIAB report ${ }^{14},(8.5$ (1.8) \%, approx. 69 (20) mmol/mol, $\mathrm{n}=993)$. We observed an inverse correlation between $\mathrm{HbA1c}$ and self-reported BG-testing frequency, as in other T1D studies ${ }^{15,16}$. Whilst in our study the average number of reported BG tests per day, 5.4 (2.7), was good, approximately one third of patients reported never testing overnight. This confirmed our hypothesis of infrequent overnight BG-testing, despite our clinicians usually recommending episodic overnight testing given the frequency and risks of $\mathrm{NH}^{4-6,8}$. Nonetheless, 
$9 \%$ of patients reported testing every night, which may indicate fear of hypoglycaemia, or perhaps caution as they live alone (data not available).

BG levels targeted are in Table 2, but with a mean HbA1c of $7.8 \%(61.3 \mathrm{mmol} / \mathrm{mol})$ the mean daily BG achieved would be $H 9.8 \mathrm{mmol} / \mathrm{l}^{17}$. Subjects targeted higher BG levels at bedtime and overnight, sometimes in the teens, to avoid NH. CSII-users targeted lower pre-bed BG levels than MDI-users, (both $>7.0 \mathrm{mmol} / \mathrm{L}$ ), but similar levels at other times. Interestingly, adults with IHA did not target higher BG levels than those reporting normal hypoglycaemia awareness.

For hypoglycaemic conscious people the then recommended (oral) treatment was $15 \mathrm{~g}$ refined carbohydrate to rapidly increase BG, followed by complex carbohydrate if the next meal was not within 20 minutes to prevent hypoglycaemia recurrence. Ideally, BG should be rechecked 15 minutes post-treatment and additional carbohydrate eaten if needed ${ }^{18}$. If consciousness is impaired glucagon injection or IV glucose administered by another person is required. Only $36 \%$ of survey patients described appropriate $\mathrm{NH}$ treatment, with $49 \%$ reporting they eat refined carbohydrate alone before returning to sleep, often without a BG test to confirm an adequate response. This could increase NH recurrence risk. Patients who consume complex carbohydrate alone may have a delayed return to normoglycaemia. We speculate this behaviour may indicate 1) need for education 2) lack of nearby optimal foods; 3) a desire to return to sleep promptly or 4) a desire to prevent post-NH hyperglycaemia through excess food. The impact of re-education on NH treatment is of interest.

A high percentage, (H50\%) of survey participants reported that they would not change their overnight diabetes plan if they had extra exercise, alcohol or illness. This is surprising as these aspects are usually part of routine diabetes education, and are common scenarios, even though people with T1D often avoid exercise due to glycaemia related challenges ${ }^{19}$. Again, whilst we believe these practices may be widespread, we could not find another relevant publication. Not 
modifying their glucose care-plan in these settings may increase patient risk of $\mathrm{NH}$ after exercise or alcohol, and of hyperglycaemia / ketoacidosis during illness. Whilst many patients desired additional diabetes education, one third of those who reported lack of confidence in adjusting their diabetes care-plan did not. Subjects reporting IHA had higher rates of (self-reported) SH, and were more likely to desire further education than those reporting normal hypoglycaemia awareness. Interestingly, education desire did not differ by diabetes duration nor insulin delivery modality, even though we speculated that CSII users may have had more recent education related to pump commencement or renewal. Our results suggest that it is difficult to predict who wants or needs additional education based on clinical factors alone, and the survey used herein may assist. The participating clinics provided additional education to subjects with unsafe behaviours reported in the survey or who requested education.

Very few patients always suggested safe responses to hypothetical bed-time BG levels. Mid-range levels, where no action was usually needed, had the highest rates of safe responses. Similar results were reported in a Type 2 diabetes (T2D) study of patients with risky glucose self-management behaviours $^{20}$. Some studies demonstrate diabetes education benefit. Bhutani et al. found improved knowledge, attitude and practices and fewer symptomatic hypoglycaemia episodes in T2D patients after structured education with their doctor ${ }^{21}$. There is substantial evidence that education is key in improving diabetes management, especially in reducing hypoglycaemia ${ }^{4,22-25}$. We found that CSII use was associated with higher rates of safer responses to hypothetical pre-bed BG levels. This may relate to more recent or additional education related to pump commencement or renewal, though their desire for further education was similar to that of MDI users. In addition, use of a bolus calculator (in insulin pumps) for insulin dose guidance was regarded as a safe choice in managing normal or high pre-bed BG levels. However, if the insulin sensitivity factor and insulin action duration are incorrect this may not be so, and also patients may not use the bolus calculator. Bolus advisors, such as in insulin pumps or "smart" BG meters, can improve insulin dose choices and 
glycaemia $^{26,27}$. Lawton et al. showed that whilst most T1D patients perceive this technology as having advantages, it also has disadvantages such as dependence on the calculator, not remembering their insulin-carbohydrate and insulin sensitivity factor(s), and that these settings can change over time ${ }^{28}$.

Study strengths are: novelty, clinical relevance, feasibility for clinical use, large sample size across two clinics and high participation rates, wide range of patient ages and diabetes duration, and analyses by insulin delivery mode, IHA status and desire for education.

Study limitations include an English-only questionnaire, risk of recall bias or reporting of ideal rather than actual behaviour and some missing data. In estimating the insulin dose to correct an elevated $\mathrm{BG}$, a target $(6 \mathrm{mmol} / \mathrm{L}$, which is a typical mean target across the day) was chosen for screening and the rule of 100 for each patient used to estimate their insulin sensitivity, which may not be accurate. NH treatment recommendations and safe behaviours may differ elsewhere, however responses in the independent clinics were similar. Only adults were studied, though with modification the survey may suit paediatric practices. BG meter downloads were not assessed to check reported testing frequency, though a practical challenge is the use of several meters. Meter records also reflect achieved BG levels, not those targeted, nor were formal questionnaires used to assess IHA, which would increase survey time and are usually a research tool. Hospital records for $\mathrm{SH}$ were not evaluated, as recall of $\mathrm{SH}$ in the prior year has been shown to be accurate and, based on our prior study, only a minority of T1D adults with a SH are transported to hospital ${ }^{29}$.

In conclusion, our new self-administered survey is feasible for clinical use and reveals that many adults with T1D/LADA have suboptimal behaviours related to their overnight glucose control. Results also show substantial knowledge or implementation gaps and many desire additional diabetes education. Additional education may improve knowledge and implementation of relevant self-care. Such a survey may be incorporated into an annual review cycle, such as associated with diabetes complication screening, and may also be used pre- and post-diabetes education. 
Acknowledgements: Authors thank study participants and other clinicians at the Diabetes Services of the Royal North Shore Hospital (Sydney) and at St. Vincent's Hospital Melbourne. For funding investigators, we thank CSN (Centrala studiestödsnämnden) (CRL) and the NHMRC (AJJ and ACK) and University of Sydney, Sydney Medical School Foundation (AJJ). This research is dedicated to my (CRL) dear friend Kristina Mood, and to all others, who like her, live with Type 1 Diabetes, with the hope that the knowledge gained will lessen the challenges. 


\section{Author Roles}

CRL - Recruited patients, collected data, managed the database, performed descriptive statistical data analysis, interpreted the data and prepared the manuscript.

ASJ - Assisted with ethics approvals, assisted with data-base design and statistical analysis and contributed to the manuscript preparation.

$\mathrm{RMcG}$ - Assisted with patient recruitment, data collection and ethics approval.

$\mathrm{JL}$ - main Swedish tutor of CRL, was involved in design of the project, discussed results and revised manuscript.

ACK - Assisted with data interpretation and manuscript preparation.

RMacI- Assisting with data collection and manuscript preparation.

GW - Assistance with patient recruitment and manuscript preparation.

DNO -Assisted with data collection, interpretation and manuscript preparation.

GRF - Assisted with data collection and interpretation, and oversaw research carried out at RNSH.

AJJ - Designed the study, including questionnaire and database, obtained ethics approvals, recruited (SVH) participants, collected data, interpreted data and helped prepare the manuscript.

All authors contributed to the manuscript and approved the final version prior to submission.

This article is protected by copyright. All rights reserved. 


\section{Conflict of interest:}

CRL - none

ASJ - none

$\mathrm{RTMcG}$ - none

$\mathrm{JL}$ - none

ACK - none

RJM - none

GMW - none

DNO - none

GRF - none

$\mathrm{AJJ}$ - none

This article is protected by copyright. All rights reserved. 


\section{References}

1. The Diabetes Control and Complications Trial Research Group. The effect of intensive treatment of diabetes on the development and progression of long-term complications in insulindependent diabetes mellitus. N Engl J Med. 1993;329(14):977-86.

2. The Diabetes Control and Complications Trial Research Group. Effect of intensive therapy on the microvascular complications of type 1 diabetes mellitus. JAMA. 2002;287(19):2563-9.

3. Bojestig M, Arnqvist HJ, Hermansson G, Karlberg BE, Ludvigsson J. Declining incidence of nephropathy in insulin-dependent diabetes mellitus. N Engl J Med. 1994;330(1):15-8.

4. International Hypoglycaemia Study Group. Minimizing Hypoglycemia in Diabetes. Diabetes care. 2015;38(8):1583-91.

5. Bergenstal RM, Klonoff DC, Garg SK, Bode BW, Meredith M, Slover RH, et al. Thresholdbased insulin-pump interruption for reduction of hypoglycemia. N Engl J Med. 2013;369(3):22432.

6. Chico A, Vidal-Rios P, Subira M, Novials A. The continuous glucose monitoring system is useful for detecting unrecognized hypoglycemias in patients with type 1 and type 2 diabetes but is not better than frequent capillary glucose measurements for improving metabolic control. Diabetes care. $2003 ; 26(4): 1153-7$.

7. Jones TW, Porter P, Sherwin RS, Davis EA, O'Leary P, Frazer F, et al. Decreased epinephrine responses to hypoglycemia during sleep. N Engl J Med. 1998;338(23):1657-62.

8. Tattersall RB, Gill GV. Unexplained deaths of type 1 diabetic patients. Diabetic Med. $1991 ; 8(1): 49-58$.

9. Vardi M, Jacobson E, Nini A, Bitterman H. Intermediate acting versus long acting insulin for type 1 diabetes mellitus. The Cochrane database of systematic reviews. 2008(3):Cd006297. 10. Benkhadra K, Alahdab F, Tamhane SU, McCoy RG, Prokop LJ, Murad MH. Continuous subcutaneous insulin infusion versus multiple daily injections in individuals with type 1 diabetes: a systematic review and meta-analysis. Endocrine. 2016;55(1):77-84. 
11. Kalra S, Mukherjee JJ, Venkataraman S, Bantwal G, Shaikh S, Saboo B, et al. Hypoglycemia: The neglected complication. Indian J Endocrin Metab. 2013;17(5):819-34.

12. Aronson R, Goldenberg R, Boras D, Skovgaard R, Bajaj H. The Canadian Hypoglycemia Assessment Tool Program: Insights Into Rates and Implications of Hypoglycemia From an Observational Study. Can J Diabetes doi: 101016/jjcjd201701007 [Epub ahead of print]. 2017.

13. Kalra S, Gupta Y. Ambulatory glucose profile: Flash glucose monitoring. JPMA J Pak Med Assoc. 2015;65(12):1360-2.

14. Flack J, Colagiuri S. Australian National Diabetes Information Audit \& Benchmarking (ANDIAB) 2011 Final Report. National Association of Diabetes Centres [cited 18 Oct 2016] [Available from: http://www.health.gov.au/internet/publications/publishing.nsf/Content/pqdiabetes-pubs-andiab11-toc].

15. Murata T, Tsuzaki K, Yoshioka F, Okada H, Kishi J, Yamada K, et al. The relationship between the frequency of self-monitoring of blood glucose and glycemic control in patients with type 1 diabetes mellitus on continuous subcutaneous insulin infusion or on multiple daily injections. J Diabetes Investig. 2015;6(6):687-91.

16. Telo GH, de Souza MS, Andrade TS, Schaan BD. Comparison between adherence assessments and blood glucose monitoring measures to predict glycemic control in adults with type 1 diabetes: a cross-sectional study. Diabetol Metab Syndr. 2016;8: 54:1-6.

17. Diabetes UK. [homepage on the Internet]. London: Diabetes UK [cited 18 Oct 2016]. Convert HbA1c to Average Blood Sugar Level 2016 [Available from: http://www.diabetes.co.uk/hba1c-to-blood-sugar-level-converter.html].

18. Kaufman FR. (ed). Medical Management of Type 1 Diabetes. 6th ed. Alexandria, VA: American Diabetes Association; 2012.

19. Brazeau AS, Rabasa-Lhoret R, Strychar I, Mircescu H. Barriers to physical activity among patients with type 1 diabetes. Diabetes Care. 2008;31(11):2108-9. 
20. Elliott JA, Abdulhadi NN, Al-Maniri AA, Al-Shafaee MA, Wahlstrom R. Diabetes selfmanagement and education of people living with diabetes: a survey in primary health care in Muscat Oman. PloS one. 2013;8(2):e57400.

21. Bhutani G, Kalra S, Lamba S, Verma PK, Saini R, Grewal M. Effect of diabetic education on the knowledge, attitude and practices of diabetic patients towards prevention of hypoglycemia. Indian J Endocrinol Metab. 2015;19(3):383-6.

22. Muhlhauser I, Jorgens V, Berger M, Graninger W, Gurtler W, Hornke L, et al. Bicentric evaluation of a teaching and treatment programme for type 1 (insulin-dependent) diabetic patients: improvement of metabolic control and other measures of diabetes care for up to 22 months.

Diabetologia. 1983;25(6):470-6.

23. Plank J, Kohler G, Rakovac I, Semlitsch BM, Horvath K, Bock G, et al. Long-term evaluation of a structured outpatient education programme for intensified insulin therapy in patients with Type 1 diabetes: a 12-year follow-up. Diabetologia. 2004;47(8):1370-5.

24. Hopkins D, Lawrence I, Mansell P, Thompson G, Amiel S, Campbell M, et al. Improved biomedical and psychological outcomes 1 year after structured education in flexible insulin therapy for people with type 1 diabetes: the U.K. DAFNE experience. Diabetes care. 2012;35(8):1638-42.

25. Elliott J, Jacques RM, Kruger J, Campbell MJ, Amiel SA, Mansell P, et al. Substantial reductions in the number of diabetic ketoacidosis and severe hypoglycaemia episodes requiring emergency treatment lead to reduced costs after structured education in adults with Type 1 diabetes. Diabet Med. 2014;31(7):847-53.

26. Parkin CG, Barnard K, Hinnen DA. Safe and Efficacious Use of Automated Bolus Advisors in Individuals Treated With Multiple Daily Insulin Injection (MDI) Therapy: Lessons Learned From the Automated Bolus Advisor Control and Usability Study (ABACUS). J Diabetes Sci Technol. 2015;9(5):1138-42.

27. Ziegler R, Cavan DA, Cranston I, Barnard K, Ryder J, Vogel C, et al. Use of an insulin bolus advisor improves glycemic control in multiple daily insulin injection (MDI) therapy patients 
with suboptimal glycemic control: first results from the ABACUS trial. Diabetes care. 2013;36(11):3613-9.

28. Lawton J, Kirkham J, Rankin D, Barnard K, Cooper CL, Taylor C, et al. Perceptions and experiences of using automated bolus advisors amongst people with type 1 diabetes: a longitudinal qualitative investigation. Diabetes Res Clin Pract. 2014;106(3):443-50.

29. Hendrieckx C, Halliday JA, Bowden JP, Colman PG, Cohen N, Jenkins A, et al. Severe hypoglycaemia and its association with psychological well-being in Australian adults with type 1 diabetes attending specialist tertiary clinics. Diabetes Res Clin Pract. 2014;103(3):430-6. 


\section{Figures and Table legends}

Figure 1. Type of treatment for nocturnal hypoglycaemia.

Footnote: Abbreviations: carbohydrate - carb; Years - yrs.

Table 1. Subject demographics

Values are mean (SD) unless otherwise specified. P-values denote significance of comparisons between continuous variables (by t-tests) and of frequency counts (by Chi-square tests).

Abbreviations: continuous subcutaneous insulin infusion - CSII; hypoglycaemia - hypo; impaired hypoglycaemia awareness - IHA; multiple daily injections - MDI; years - yrs

Table 2. Glucose testing habits, targets and hypoglycaemia frequency

Values are mean (SD) or \%, unless otherwise specified. The table shows self-reported BG levels targeted (not achieved) at various times of the day, the frequency of BG testing at various times of the day, and hypoglycaemia frequency during the day and overnight, including SH in the last year. Values are shown for all subjects, and stratified by insulin delivery mode, IHA status and further education desire. P-values denote significance of comparisons between continuous variables ( $t$ tests) and of frequency counts (Chi-square tests).

Abbreviations: blood glucose - BG; continuous subcutaneous insulin infusion - CSII; education educ'n; hypoglycaemia - hypo; impaired hypoglycaemia awareness - IHA; multiple daily injections - MDI

Table 3. Percentage of safe hypothetical pre-bed BG treatment answers

The table outlines the percentage of safe answers given by all participants, and as divided by insulin delivery mode, IHA status, and by desire for further diabetes education. As indicated with an asterisk *, there were significant differences: between CSII and MDI users for scenarios with BG 8, 18 (with moderate ketones) and $20 \mathrm{mmol} / \mathrm{l}$; by IHA status in the BG $8 \mathrm{mmol} / \mathrm{l}$ scenario. Overall 
CSII-users suggested safer responses more often than MDI-users, $p=0.0003$. There was no difference between subjects with regard to education desire.

Abbreviations: continuous subcutaneous insulin infusion - CSII; hypoglycaemia - hypo; impaired hypoglycaemia awareness - IHA; multiple daily injections - MDI 
Figure 1.

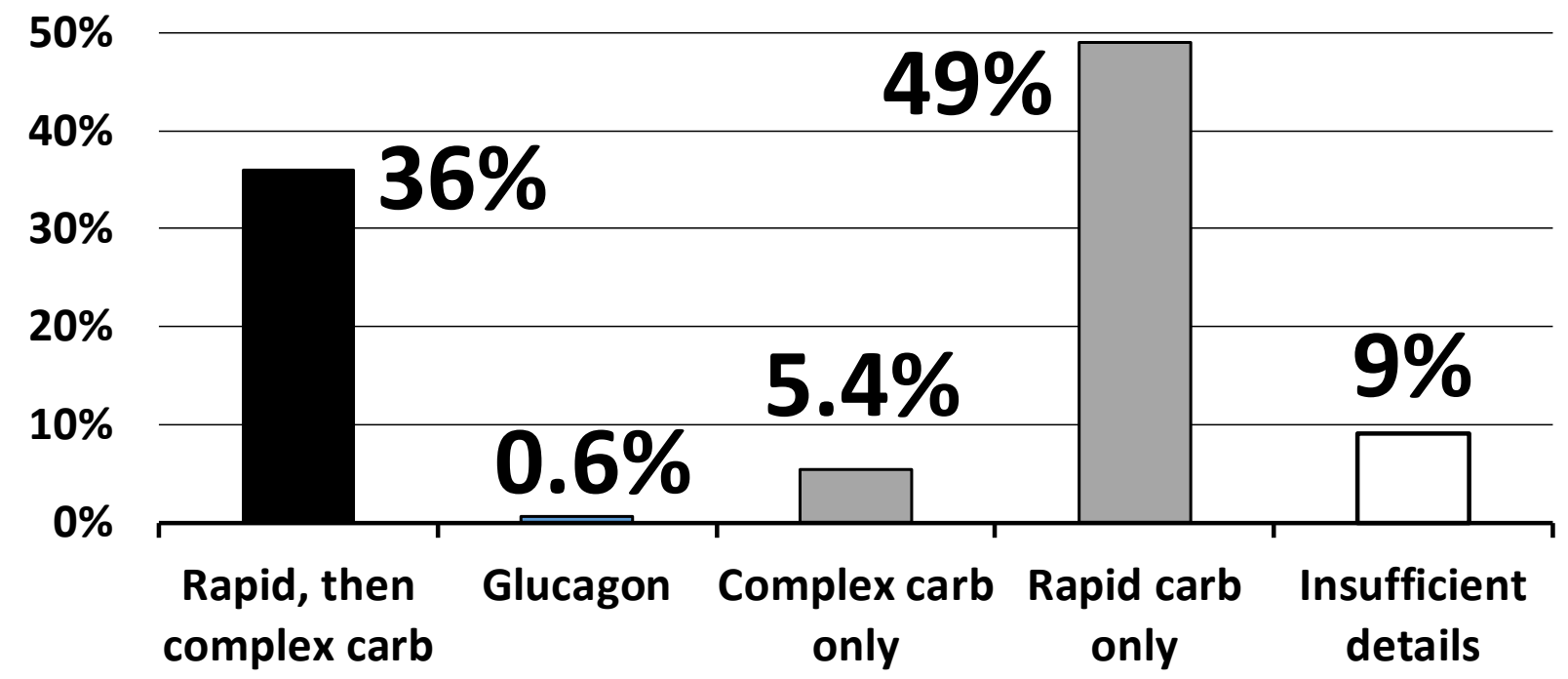


Table 1

\begin{tabular}{|c|c|c|c|c|c|c|c|c|c|c|}
\hline & \multirow{2}{*}{ Total } & \multirow{2}{*}{ CSII } & \multirow{2}{*}{ MDI } & \multirow{2}{*}{$\mathbf{p}$} & \multirow{2}{*}{ Hypo aware } & \multirow{2}{*}{ IHA } & \multirow{2}{*}{$\mathbf{p}$} & \multicolumn{3}{|c|}{$\begin{array}{c}\text { Desires more diabetes } \\
\text { education }\end{array}$} \\
\hline & & & & & & & & Yes & No & $\mathbf{p}$ \\
\hline $\mathbf{n}$ & 205 & 77 & 128 & - & 131 & 74 & - & 65 & 140 & \\
\hline Age (yrs) & $41(17)$ & $41(17)$ & $41(17)$ & 0.96 & $37(16)$ & $47(17)$ & 0.0001 & $44(17)$ & $39(17)$ & 0.08 \\
\hline Gender (\%male) & 50 & 34 & 59 & 0.0004 & 48 & 53 & 0.53 & 48 & 50 & 0.72 \\
\hline Yrs T1D & $20(16)$ & $20(14)$ & $20(17)$ & 0.81 & $17(13)$ & $27(18)$ & $<0.0001$ & $21(17)$ & $20(15)$ & 0.64 \\
\hline HbA1c (\%) & $7.8(1.4)$ & $7.8(1.1)$ & $7.7(1.6)$ & 0.50 & $7.7(1.3)$ & $7.8(1.7)$ & 0.83 & $8.0(1.9)$ & $7.6(1.2)$ & 0.17 \\
\hline HbA1c (mmol/mol) & $62(8)$ & $62(12)$ & $61(18)$ & 0.50 & $61(14)$ & $62(19)$ & 0.83 & $64(21)$ & $60(13)$ & 0.17 \\
\hline$\%$ on CSII & 38 & 100 & 0 & - & 36 & 23 & 0.51 & 35 & 38 & 0.71 \\
\hline
\end{tabular}

This article is protected by copyright. All rights reserved. 
Table 2.

\begin{tabular}{|c|c|c|c|c|c|c|c|c|c|c|}
\hline \multicolumn{11}{|c|}{ (Self-reported) Blood glucose target } \\
\hline 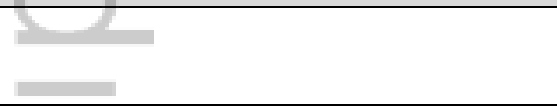 & Total & CSII & MDI & $\mathbf{p}$ & Hypo aware & IHA & $\mathbf{p}$ & $\begin{array}{c}\text { Educ'n } \\
\text { Yes }\end{array}$ & $\begin{array}{c}\text { Educ'n } \\
\text { No }\end{array}$ & $\mathbf{p}$ \\
\hline Pre-breakfast & $6.5(1.2)$ & $6.5(1.0)$ & $6.5(1.3)$ & 0.86 & $6.5(1.2)$ & $6.6(1.2)$ & 0.61 & $6.7(1.1)$ & $6.4(1.2)$ & 0.09 \\
\hline Pre-lunch & $7.0(1.3)$ & $6.9(1.3)$ & $7.0(1.3)$ & 0.46 & $7.0(1.2)$ & $6.9(1.4)$ & 0.59 & $7.0(1.3)$ & $6.9(1.3)$ & 0.63 \\
\hline After Lunch & $6.9(1.3)$ & $6.7(1.2)$ & $7.0(1.3)$ & 0.11 & $7.0(1.3)$ & $6.7(1.2)$ & 0.07 & $7.0(1.2)$ & $6.8(1.3)$ & 0.36 \\
\hline Pre-dinner & $7.1(1.3)$ & $6.9(1.1)$ & $7.2(1.4)$ & 0.06 & $7.1(1.2)$ & $7.0(1.5)$ & 0.55 & $7.2(1.4)$ & $7.0(1.3)$ & 0.47 \\
\hline Overall Daytime & $6.9(1.0)$ & $6.7(1.0)$ & $6.9(1.0)$ & 0.16 & $6.9(1.0)$ & $6.8(1.1)$ & 0.46 & $7.0(0.9)$ & $6.8(1.1)$ & 0.20 \\
\hline Pre-bedtime & $7.5(1.4)$ & $7.1(1.2)$ & $7.7(1.4)$ & 0.004 & $7.6(1.4)$ & $7.3(1.3)$ & 0.23 & $7.7(1.1)$ & $7.4(1.5)$ & 0.17 \\
\hline Overnight & $7.1(1.3)$ & $7.0(1.1)$ & $7.2(1.4)$ & 0.17 & $7.2(1.4)$ & $7.0(1.3)$ & 0.47 & $7.3(1.0)$ & $7.0(1.4)$ & 0.12 \\
\hline \multicolumn{11}{|c|}{ (Self-reported) Blood glucose testing frequency } \\
\hline BG tests / day Mean (SD) & $5.4(2.7)$ & $6.0(2.9)$ & $5.1(2.4)$ & $\mathbf{0 . 0 2}$ & $5.3(2.6)$ & $5.7(2.8)$ & 0.26 & $5.9(3.0)$ & $5.3(2.5)$ & 0.17 \\
\hline $\begin{array}{c}\text { Overnight BG testing (\%) } \\
\text { (never / sometimes / } \\
\text { every night) }\end{array}$ & $27 / 64 / 9$ & $19 / 68 / 13$ & $32 / 62$ / 6 & 0.07 & $29 / 60 / 11$ & $25 / 70 / 5$ & 0.30 & 24 / 65 / 11 & $29 / 63 / 8$ & 0.63 \\
\hline \multicolumn{11}{|c|}{ (Self-reported) Hypoglycaemia frequency } \\
\hline $\begin{array}{c}\text { Day time hypos / week (\%) } \\
(<1 / 1-3 />3 \text { times })\end{array}$ & $41 / 40 / 19$ & $29 / 45$ / 26 & $48 / 37 / 15$ & 0.02 & $43 / 43 / 14$ & $36 / 35 / 28$ & 0.04 & $38 / 35 / 26$ & $42 / 42 / 16$ & 0.21 \\
\hline $\begin{array}{l}\text { Overnight hypos / month }(\%) \\
\text { (never / 1-4 / > } 4 \text { times) }\end{array}$ & $21 / 65 / 13$ & $16 / 66 / 18$ & $25 / 65 / 10$ & 0.13 & $18 / 67 / 15$ & $27 / 62 / 11$ & 0.35 & $16 / 68 / 16$ & $24 / 64 / 12$ & 0.45 \\
\hline SH (\%) (in last yr) & 21 & 23 & 20 & 0.51 & 12 & 36 & $<0.0001$ & 25 & 19 & 0.33 \\
\hline
\end{tabular}

This article is protected by copyright. All rights reserved. 
Table 3.

\begin{tabular}{|c|c|c|c|c|c|c|c|}
\hline & \multicolumn{6}{|c|}{ Hypothetical Pre-Bed BG \pm ketones scenario } & \\
\hline mmol/L & $\mathbf{4}$ & $\mathbf{8}$ & $\mathbf{1 1}$ & $\mathbf{1 5}$ & $\begin{array}{c}\mathbf{1 8}+ \\
\mathbf{1 . 0} \text { ketones }\end{array}$ & $\mathbf{2 0}$ & Overall \\
\hline Safe (\%) - All & 54 & 87 & 95 & 47 & 70 & 63 & 28 \\
\hline Safe (\%) - CSII users & 62 & $\mathbf{9 4}$ & 97 & 53 & $\mathbf{7 9 *}$ & $\mathbf{7 3 *}$ & $\mathbf{4 3}$ \\
\hline Safe (\%) - MDI users & 48 & $\mathbf{8 3} *$ & 94 & 43 & $\mathbf{6 4}^{*}$ & $\mathbf{5 7 *}$ & $\mathbf{2 0}$ \\
\hline Safe (\%) - Hypo aware & 54 & $\mathbf{8 2 *}$ & 96 & 47 & 72 & 62 & 27 \\
\hline Safe (\%) - IAH & 53 & $\mathbf{9 5} *$ & 93 & 47 & 66 & 64 & 30 \\
\hline Safe (\%) - Desires education & 60 & 91 & 94 & 47 & 68 & 62 & 32 \\
\hline Safe (\%) - No desire for education & 51 & 85 & 96 & 47 & 72 & 64 & 27 \\
\hline
\end{tabular}




\section{University Library}

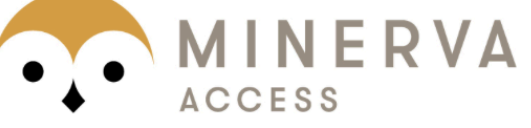

A gateway to Melbourne's research publications

Minerva Access is the Institutional Repository of The University of Melbourne

\section{Author/s:}

Larsson, CR;Januszewski, AS;McGrath, RT;Ludvigsson, J;Keech, AC;Maclsaac, RJ;Ward, GM;O'Neal, DN;Fulcher, GR;Jenkins, AJ

Title:

Suboptimal behaviour and knowledge regarding overnight glycaemia in adults with type 1 diabetes is common

Date:

2018-09-01

Citation:

Larsson, C. R., Januszewski, A. S., McGrath, R. T., Ludvigsson, J., Keech, A. C., Maclsaac, R. J., Ward, G. M., O'Neal, D. N., Fulcher, G. R. \& Jenkins, A. J. (2018). Suboptimal behaviour and knowledge regarding overnight glycaemia in adults with type 1 diabetes is common. INTERNAL MEDICINE JOURNAL, 48 (9), pp.1080-1086. https://doi.org/10.1111/ imj.13798.

Persistent Link:

http://hdl.handle.net/11343/283775 